\title{
Inconsistent classification of pusher behaviour in stroke patients: a direct comparison of the Scale for Contraversive Pushing and the Burke Lateropulsion Scale
}

Clinical Rehabilitation 2014, Vol. 28(7) 696-703

(C) The Author(s) 2014

Reprints and permissions: sagepub.co.uk/journalsPermissions.nav DOI: I0.1 I77/02692I55।3517726 cre.sagepub.com

@SAGE

\author{
Jeannine Bergmann 1,2, Carmen Krewer ${ }^{1,2}$, \\ Katrin Rieß ${ }^{1,2}$, Friedemann Müller ${ }^{1,2}$, \\ Eberhard Koenig ${ }^{1,2}$ and Klaus Jahn, 2,3
}

\begin{abstract}
Objective: To compare the classification of two clinical scales for assessing pusher behaviour in a cohort of stroke patients.

Design: Observational case-control study.

Setting: Inpatient stroke rehabilitation unit.

Subjects: A sample of 23 patients with hemiparesis due to a unilateral stroke ( $1.6 \pm 0.7$ months post stroke). Methods: Immediately before and after three different interventions, the Scale for Contraversive Pushing and the Burke Lateropulsion Scale were applied in a standardized procedure.

Results: The diagnosis of pusher behaviour on the basis of the Scale for Contraversive Pushing and the Burke Lateropulsion Scale differed significantly $(\chi 2=54.260, p<0.00 \mathrm{I})$ resulting in inconsistent classifications in $3 \mathrm{I}$ of 138 cases. Changes immediately after the interventions were more often detected by the Burke Lateropulsion Scales than by the Scale for Contraversive Pushing $(\chi 2=19.148, p<0.001)$. All cases with inconsistent classifications showed no pusher behaviour on the Scale for Contraversive Pushing, but pusher behaviour on the Burke Lateropulsion Scale. 64.5\% (20 of 31 ) of them scored on the Burke Lateropulsion Scale on the standing and walking items only.

Conclusions: The Burke Lateropulsion Scale is an appropriate alternative to the widely used Scale for Contraversive Pushing to follow-up patients with pusher behaviour (PB); it might be more sensitive to detect mild pusher behaviour in standing and walking.
\end{abstract}

\section{Keywords}

Stroke, pusher syndrome, Scale for Contraversive Pushing, Burke Lateropulsion Scale

Received: 8 July 2013; accepted: 30 November 2013

\footnotetext{
ISchoen Klinik Bad Aibling, Germany

${ }^{2}$ German Center for Vertigo and Balance Disorders (DSGZ), Ludwig-Maximilians University of Munich, Germany

${ }^{3}$ Department of Neurology, Ludwig-Maximilians University of Munich, Germany
}

\section{Corresponding author:}

Jeannine Bergmann, Schön Klinik Bad Aibling, Kolbermoorer Strasse 72, Bad Aibling, D-83043, Germany.

Email: JBergmann@Schoen-Kliniken.de 


\section{Introduction}

Pusher behaviour is characterized by an active lateral tilt of the body and resistance to passive correction of the tilted posture. ${ }^{1}$ Patients with pusher behaviour show an erroneous internal reference of verticality. ${ }^{2,3}$ This leads to a shift of the centre of gravity toward the paretic side and can result in loss of balance and falls. ${ }^{4,5}$

Pusher behaviour is very relevant in stroke rehabilitation, because it prolongs inpatient treatment. ${ }^{6}$ However, there is much uncertainty about its prevalence, what may be due to heterogeneous diagnostic criteria. ${ }^{7-9}$

Clinical scales have been proposed for the diagnosis of pusher behaviour, e.g. the Scale for Contraversive Pushing and the Burke Lateropulsion Scale. Both scales reflect criteria set out by Davies ${ }^{1}$ to distinguish patients with pusher behaviour; however, classification based on these scales might be inconsistent, for they show great variations in the selection of items and the scoring. The Scale for Contraversive Pushing rates the degree of postural symmetry, the presence of abduction or extension of the non-paretic extremities, and the presence of resistance to passive correction. The Burke Lateropulsion Scale assesses the degree of action or reaction of the patients to keep or change a position. It is the only scale that incorporates pusher behaviour in supine rolling and in walking. The clinimetric properties and the clinical applicability of the two scales were recently reviewed by Babyar et al. ${ }^{10}$ While the Scale for Contraversive Pushing is more extensively evaluated, the Burke Lateropulsion Scale also shows evidence of clinical and research practicability. $7,11,12$

Consistent measures are urgently needed to identify and follow-up pusher behaviour. They are a prerequisite for studying the epidemiology, the underlying mechanisms, prognostic factors, and the effectiveness of therapies.

The aim of our study was to compare the classifications of pusher behaviour based on the Scale for Contraversive Pushing and the Burke Lateropulsion Scale in the same sample of stroke patients.

\section{Methods}

The present study is a secondary analysis of a cross-over study on the effects of different therapeutic interventions on pusher behaviour. The methods and primary results of the study were reported in detail elsewhere. ${ }^{13}$

\section{Patients}

Patients with hemiparesis due to a unilateral hemispheric stroke were enrolled in the study. Additional inclusion criteria were age $\geq 18$ years, inability to stand unassisted, but previous ability to walk independently before stroke. Exclusion criteria due to therapeutic interventions were body weight above $150 \mathrm{~kg}$, body height below 1.60 meters and above 1.90 meters, unstable cardiac disease, metal implants, brain tumour, meningitis, epilepsy, vestibular disorders, eye muscle paralysis, neurodegenerative movement disorder, unstable fracture, severe osteoporosis, contractures or spasticity of the lower extremities.

The Ethics Committee of the LudwigMaximilians University Munich approved the study in accordance with the Declaration of Helsinki. Written informed consent was given by all patients or their legal representatives.

\section{Assessments and procedure}

The Scale for Contraversive Pushing includes three components: (1) the symmetry of spontaneous body posture (rated with $0,0.25,0.75$, or 1 point), (2) the use of non-paretic extremities $(0,0.5$, or 1 point), and (3) the resistance to passive correction of the tilted posture ( 0 or 1 point). ${ }^{3,14}$ Each component is tested in sitting and standing position, yielding a maximum score of 2 per component. For a diagnosis of pusher behaviour all three components must be present. Karnath et al. ${ }^{3}$ originally recommended a cut-off score equal to or greater than one (cut-off $\geq 1$ ) for each component (sitting plus standing). A less conservative cut-off score greater than zero (cut-off $>0$ ) for each component was evaluated by Baccini et al. ${ }^{7,12}$, who found improved diagnostic accuracy.

The Burke Lateropulsion Scale assesses the patient's resistance to passive supine rolling, to passive postural correction when sitting and standing, and to assistance during transferring and walking. ${ }^{11}$ The score for each item is rated on a scale from 0 to 3 ( 0 to 4 for standing) and is based on the severity of resistance or the tilt angle when the 
patients starts to resist the passive movement. The cut-off for the diagnosis of pusher behaviour is $\geq 2$ points. ${ }^{10}$

Standardized frontal photographs were made to study postural responses and compare them to the items of the clinical scales. Head, trunk, and leg orientation were measured in three positions: spontaneous sitting on the physiotherapist's bench with feet having ground contact, spontaneous sitting with legs hanging freely, and standing. Detailed instruction can be found in the supplementary material appendix.

Patients in the study underwent three different therapeutic interventions in a pseudo-random order over 1 week. Immediately before and after each therapy, the Scale for Contraversive Pushing and the Burke Lateropulsion Scale were assessed by the same blinded and trained examiner, and standardized photographs were taken. The following assessment sequence was defined to apply the measures in a single procedure: assisted transfer from the wheelchair to the therapy bench toward the nonparetic side (relevant for the Scale for Contraversive Pushing, B-sitting and the Burke Lateropulsion Scale, transfer), supine rolling (Burke Lateropulsion Scale, supine), sitting on the bench with the feet having ground contact and the knees at a $90^{\circ}$ angle (Scale for Contraversive Pushing, A-sitting and photograph), passive correction of the body position (Scale for Contraversive Pushing, C-sitting), sitting on the bench without feet having ground contact and hands in the lap (photograph), passive tilting to the paretic and non-paretic side (Burke Lateropulsion Scale, sitting), assisted standing (Scale for Contraversive Pushing, A-standing and photograph), assisted standing with passive tilting and correction (Scale for Contraversive Pushing, C-standing and Burke Lateropulsion Scale, standing), assisted walking (Burke Lateropulsion Scale, walking) and transfer via stance and toward the paretic side back into the wheelchair (Scale for Contraversive Pushing, B-walking).

\section{Statistics}

The chi-square test and Cohen's kappa coefficient $(k)$ were calculated for the classification of pusher behaviour and the detection of changes to estimate the agreement between the Scale for Contraversive Pushing and the Burke Lateropulsion Scale.

For comparisons of the leg, trunk, and head position between groups, ANOVAs were performed and posthoc Bonferroni tests were applied.

Data were analyzed with the statistical package IBM SPSS Statistics 19. The statistical $\alpha$-level was set at 0.05 .

\section{Results}

Twenty-three patients with unilateral hemispheric stroke were enrolled in the study (mean age $68 \pm 10$ years; 6 females; 19 right brain hemisphere damaged; $1.6 \pm 0.7$ months post stroke). Ten of them were classified as pushers by the Scale for Contraversive Pushing at first study visit (Scale for Contraversive Pushing score $3.25 \pm 2.00$ (median \pm interquartile range), Burke Lateropulsion Scale score $7.5 \pm 4.0$ ). Immediately before and after each of the three therapeutic interventions the data was assessed, resulting in a total of 138 data sets.

The diagnoses of pusher behaviour based on the Scale for Contraversive Pushing (cut-off $>0$ ) and the Burke Lateropulsion Scale are shown in Table $1(\chi 2(1)=54.260, p<0.001 ; k=0.564, S E$ $=0.062)$. They resulted in an inconsistent classification for 31 data sets, which originated from nine patients.

A comparison of the original and the modified Scale for Contraversive Pushing cut-off scores revealed that the original cut-off $(\geq 1)$ missed pusher behaviour in two cases compared to the modified cut-off $(>0)$. For further analysis, the cutoff $>0$ was used.

The Scale for Contraversive Pushing was taken as reference standard to calculate the sensitivity and specificity of the Burke Lateropulsion Scale, which resulted in $100 \%$ and $67 \%$, respectively.

Changes were estimated as difference between the scores immediately before and after a therapeutic intervention. The number of detected changes is shown in Table 2 and significantly differed between the Scale for Contraversive Pushing and the Burke Lateropulsion Scale $(\chi 2(1)=19.148, p<0.001)$ and showed moderate agreement $(k=0.500, S E=$ 0.103 ). 
Table I. Classification of pusher behavior based on the Scale for Contraversive Pushing and the Burke Lateropulsion Scale.

\begin{tabular}{lllr}
\hline Burke Lateropulsion Scale & \multicolumn{2}{l}{ Scale for Contraversive Pushing (cut-off $>0$ ) } & Total \\
\cline { 2 - 4 } & Pusher behaviour & No pusher behaviour & 75 \\
\hline Pusher behaviour & 44 & 31 & 63 \\
No pusher behaviour & 0 & 63 & 138 \\
Total & 44 & 94 & \\
\hline
\end{tabular}

Table 2. Changes of pusher behaviour detected on the Scale for Contraversive Pushing and the Burke Lateropulsion Scale.

\begin{tabular}{llll}
\hline Burke Lateropulsion Scale & \multicolumn{2}{l}{ Scale for Contraversive Pushing } & Total \\
\cline { 2 - 4 } & Change & No change & 29 \\
\hline Change & 16 & 13 & 40 \\
No change & 3 & 37 & 69 \\
Total & 19 & 50 & 0 \\
\hline
\end{tabular}

According to the classifications based on the Scale for Contraversive Pushing and the Burke Lateropulsion Scale, data sets were divided into a group with consistently positive diagnosis of pusher behaviour $\left(\mathrm{PB}^{+/+}\right)$, a group with inconsistent diagnosis of pusher behaviour $\left(\mathrm{PB}^{-/+}\right)$, and a group with a consistently negative diagnosis $\left(\mathrm{PB}^{-/}\right)$.

All 31 cases of $\mathrm{PB}^{-/+}$were classified as pushers on the Burke Lateropulsion Scale, but not on the Scale for Contraversive Pushing. For these cases the item scores were examined. $\mathrm{PB}^{-/+}$showed signs of pusher behaviour mostly in the standing items: 27 of 31 cases showed no points on the Scale for Contraversive Pushing components in sitting and 25 cases no points on the Burke Lateropulsion Scale sitting items. In standing 23 of 31 cases scored on the Scale for Contraversive Pushing component A (symmetry of body posture), 13 cases on the component $\mathrm{C}$ (resistance to correction), but only three cases on the component B (use of non-paretic extremities).

In seven of 31 cases no points were scored on the Scale for Contraversive Pushing, neither in sitting nor in standing, however all of them, except one case, scored only on the Burke Lateropulsion Scale standing and walking items. Regarding the entire $\mathrm{PB}^{-/+}$group, even 20 cases scored on the
Burke Lateropulsion Scale on the standing and walking items only.

The values of the head, trunk, and non-paretic leg positions determined by photographs, and the results of the ANOVAs and the posthoc comparisons are shown in Table 3.

\section{Discussion}

The Scale for Contraversive Pushing and the Burke Lateropulsion Scale showed moderate agreement in the diagnosis of pusher behaviour with higher sensitivity but lower specificity for the Burke Lateropulsion Scale in comparison to the Scale for Contraversive Pushing. The scales resulted in inconsistent classifications in patients with mild or resolving pusher behaviour. In these patients the Burke Lateropulsion Scale might be especially useful to detect pusher behaviour in standing and walking.

The Scale for Contraversive Pushing and the Burke Lateropulsion Scale resulted in $22.5 \%$ of cases in inconsistent classifications. In all these cases, the Burke Lateropulsion Scale diagnosed pusher behaviour but the Scale for Contraversive Pushing did not. 


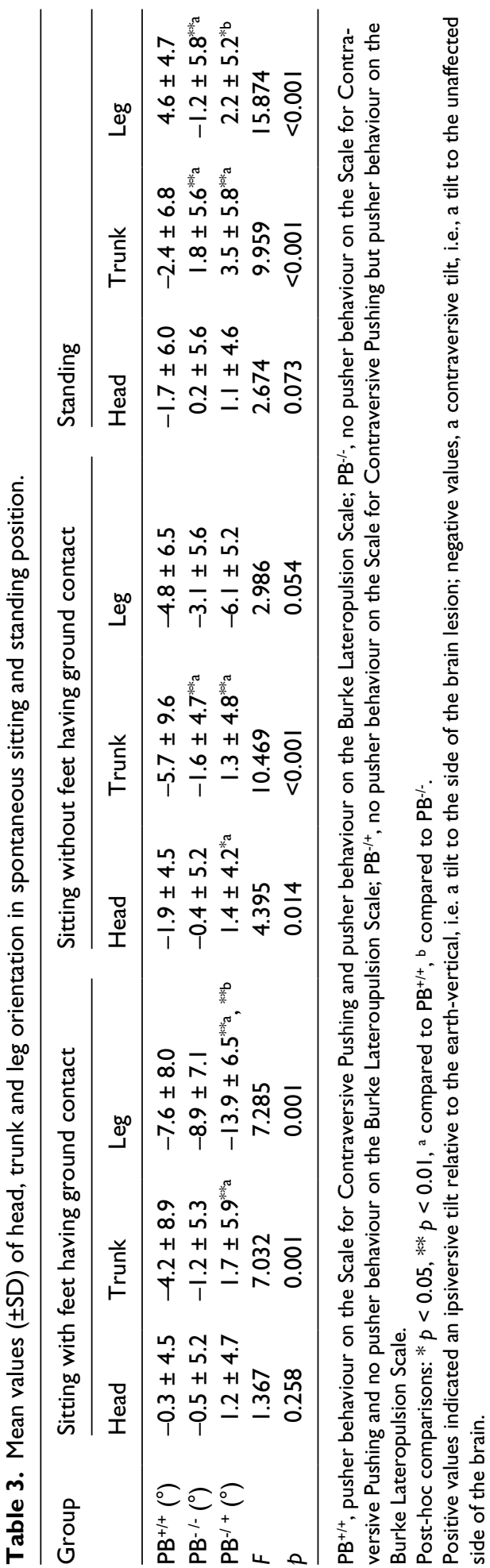

When the original cut-off criterion of the Scale for Contraversive Pushing was used, which has been suggested by Karnath et al. ${ }^{3}$, two more cases were inconsistently classified. Baccini et al. ${ }^{12}$ found an excellent agreement between the cut-off $>0$ and the clinical diagnosis of pusher behaviour, whereas the original cut-off failed to detect pusher behaviour in patients with slight symptoms. Even with the cut-off $>0$, all signs described by Davies ${ }^{1}$ must be present for the diagnosis of pusher behaviour. Consequently, we recommend the use of the cut-off $>0$ and refer to it in the following discussion.

Since there is no gold standard for the diagnosis of pusher behaviour and proof of validity was not carried out by an expert rating, we calculated the sensitivity and the specificity of the Burke Lateropulsion Scale compared to the Scale for Contraversive Pushing. The Burke Lateropulsion Scale has a higher sensitivity but a lower specificity than the Scale for Contraversive Pushing for detecting pusher behaviour and might produce more false-negative diagnoses.

We also found the Burke Lateropulsion Scale to be more responsive to small changes than the Scale for Contraversive Pushing. This supports the suggestion of Babyar et al. ${ }^{10}$ that the Burke Lateropulsion Scale might be more useful for monitoring patients with pusher behaviour as well as for assessing small changes in their status. The clinical relevance of the detected changes is not yet clear; however, small improvements are important for the rehabilitation process and might facilitate the mobilisation and therapy of the patients. In a recent study, Clark et al. ${ }^{15}$ showed that the Burke Lateropulsion Scale can be used to monitor progress and recovery during rehabilitation. The wider range of the Burke Lateropulsion Scale allows a more differentiated and graduated evaluation of pusher behaviour; the scale can be used to grade the severity of pusher behaviour across the full continuum of scores and reflects the progress most patients make during rehabilitation. ${ }^{10}$

The cases inconsistently classified by the Burke Lateropulsion Scale and the Scale for Contraversive Pushing $\left(\mathrm{PB}^{-/+}\right)$, showed signs of pusher behaviour mainly in the standing but not in the sitting items. 
$64.5 \%$ of them scored on the Burke Lateropulsion Scale on the standing and walking items only. These two items seem to be crucial for the inconsistent classification between the scales. While walking is not included in the Scale for Contraversive Pushing, both scales address resistance in standing. However, the Scale for Contraversive Pushing rates resistance to an upright position, whereas the Burke Lateropulsion Scale additionally determines resistance to moving the patient 10 degrees past midline. Resistance past midline is only measured in standing and scored with one point. Thus standing is the only item rated on a scale from 0 to 4 . The authors established this weighting to emphasise features thought to be most characteristic of pusher behaviour. ${ }^{11}$

Another important difference between the two scales, is that the Burke Lateropulsion Scale rates exclusively resistance to passive correction through a larger variety of postures (lying, sitting, standing, transferring, and walking), while the Scale for Contraversive Pushing addresses resistance in only one component in sitting and standing, respectively. On the Burke Lateropulsion Scale resistance is scored on a scale from 0 to 3 (0 to 4 for standing), on the Scale for Contraversive Pushing, however, according to the 'all-or-nothing' principle, with either 1 point (resistance is shown) or 0 points (resistance is not shown).

As mentioned, the walking item of the Burke Lateropulsion Scale is very relevant for the inconsistent classification of the two scales. However, we observed some difficulties in the assessment of this item. All patients included in this study were not able to stand unassisted and most of them needed either a lot of assistance to walk or were not able to walk at all. Consequently, the walking item was very difficult for severely impaired patients to do and for the examiner to rate. The authors of the scale recommended that, if it is not possible to assess patients in standing or walking due to marked lateropulsion they should be scored as having maximum deficit for those tasks that could not be tested. ${ }^{11}$ However, it was not always evident during the assessment of the scales in our study, if standing and walking were impossible due to the severity of lateropulsion or due to other impairments.
Like Clark et al. ${ }^{15}$ we also had problems in detecting small body tilts or determining the degree of tilt in the sitting and standing items of the Burke Lateropulsion Scale. In our study, the examiner was on the paretic side and assisted the patient while assessing the scales. It might be useful to have the examiner in front of the patient to judge deviation from verticality and responses of the trunk or the limbs. However, at the same time, the examiner has to move the patient and feel the potential resistance against the movement. Standardized photographs of the patient in a frontal view might help identifying body tilts that the examiner has difficulties detecting while sitting or standing on the patient's side.

When we compared the item scores and the body positions determined by photographs the following was evident: patients of $\mathrm{PB}^{-/+}$scored only three times on the Scale for Contraversive Pushing component $\mathrm{B}$ in standing, but more than $74 \%$ scored on the component $\mathrm{A}$, i.e., abduction of the non-paretic leg was rarely observed, but in many cases a contraversive body tilt. In contrast, data of the photographs revealed abduction of the non-paretic leg and an average slightly ipsiversively tilted trunk position. This indicates that, although these patients were able to bring their upper body to an upright position or even past midline, they were unable to place their centre of gravity over the base of support in standing. Also in sitting, photographs revealed a noticeable tilt of the non-paretic leg in the $\mathrm{PB}^{-/+}$group. Despite the clinical scales detected no pusher behaviour, the postural responses of these patients seem not completely recovered. Further research is needed to improve the understanding of the mechanism behind pusher behaviour and its recovery process.

Summing up, the Burke Lateropulsion Scale seems to be an appropriate alternative to the widely used Scale for Contraversive Pushing and especially useful to detect patients with very mild pusher behaviour and to track small changes in the behaviour. However, until now, there are no data available on sensitivity, specificity, and internal consistency of the scale. Concurrent validity was estimated by correlating the lateropulsion score with the Fugl-Meyer Balance score and the FIM motor score. ${ }^{11}$ Patients are thought to show pusher 
behaviour when scoring two or greater on the scale, but this cut-off value has not been validated to our knowledge and is inconsistently used in the literature. Babyar et al. ${ }^{10,16}$ applied the cut-off $\geq 2$, while Clark et al. ${ }^{15}$ in a recent study used the cut-off $>2$. In our sample, a cut-off value $>2$ instead of $\geq 2$ improves the agreement between the scales from $77.5 \%$ (107 of 138$)$ to $85.5 \%$ (118 of 138). As there is no gold standard for the diagnosis of pusher behaviour, a validation of the cut-off score against postural abnormalities or the subjective postural vertical might be meaningful.

There are some limitations to this study, including the small number of patients $(n=23)$. However, this number is comparable to other studies investigating patients with pusher behaviour and the total number of analyzed data sets is quite high as six measurements per patient were included. ${ }^{3,12}$ At the same time, the repeated measurement design could be a limitation of the study, since each measurement was analyzed as independent measure for comparison of classifications, what might have biased the result. We performed another chi-square test for comparison of classification including only the data at first study visit and the test was highly significant $(p<0.001)$. Thus, the repeated measurements do not seem to significantly distort our results.

A limitation with regard to the photographs is the dependency on the angulation of the focal point of the camera. We tried to minimise this bias by using a standardised protocol. Furthermore, body orientation was only determined in the frontal plane and deviations in the horizontal or sagittal plane were not taken into account.

\section{Clinical messages}

- The Burke Lateropulsion Scale is more responsive to small changes and more sensitive in the classification of pusher behaviour than the Scale for Contraversive Pushing.

- The Burke Lateropulsion Scale is especially useful to detect mild or resolving pusher behaviour in standing and walking.

\section{Contributors}

JB recruited the patients, collected the data, performed data analysis and interpretation of data and wrote the paper. CK designing the study, performed acquisition and interpretation of data and revised the paper. KR designed the study, performed acquisition of data and revised the paper. FM, EK and KJ contributed to the conception of the study and the interpretation of data analysis and revised the paper.

\section{Acknowledgements}

The authors would like to thank G Waritschlager for performing the neuroorthoptic examinations, S Heller and the team of physiotherapists for assisting with therapies, and $\mathrm{J}$ Benson for copyediting the manuscript.

\section{Conflict of interest}

The authors declare that there is no conflict of interest.

\section{Funding}

This work was supported by funds from the German Federal Ministry of Education and Research (BMBF IFB 01EO0901).

\section{References}

1. Davies PM. Out of line (The pusher syndrome). In: Steps to follow a guide to the treatment of adult hemiplegia. 1st ed. Berlin: Springer, 1985, pp. 266-284.

2. Pérennou DA, Mazibrada G, Chauvineau V, et al. Lateropulsion, pushing and verticality perception in hemisphere stroke: a causal relationship? Brain 2008; 131: 2401-2413.

3. Karnath HO, Ferber S and Dichgans J. The origin of contraversive pushing: evidence for a second graviceptive system in humans. Neurology 2000; 55: 1298-1304.

4. Karnath $\mathrm{HO}$ and Broetz D. Understanding and treating "pusher syndrome". Phys Ther 2003; 83: 1119-1125.

5. Lafosse C, Kerckhofs E, Vereeck L, et al. Postural abnormalities and contraversive pushing following right hemisphere brain damage. Neuropsychol Rehabil 2007; 17 : 374-396.

6. Krewer C, Luther M, Müller F and Koenig E. Time course and influence of pusher behavior on outcome in a rehabilitation setting: a prospective cohort study. Top Stroke Rehabil 2013; 20: 331-339.

7. Baccini M, Paci M, Nannetti L, Biricolti C and Rinaldi LA. Scale for contraversive pushing: cutoff scores for diagnosing "pusher behavior" and construct validity. Phys Ther 2008; 88: 947-955. 
8. Danells CJ, Black SE, Gladstone DJ and McIlroy WE. Poststroke "pushing": natural history and relationship to motor and functional recovery. Stroke 2004; 35 : 2873-2878.

9. Pedersen PM, Wandel A, Jorgensen HS, et al. Ipsilateral pushing in stroke: incidence, relation to neuropsychological symptoms, and impact on rehabilitation. The Copenhagen Stroke Study. Arch Phys Med Rehabil 1996; 77: 25-28.

10. Babyar SR, Peterson MG, Bohannon R, et al. Clinical examination tools for lateropulsion or pusher syndrome following stroke: a systematic review of the literature. Clin Rehabil 2009; 23: 639-650.

11. D'Aquila MA, Smith T, Organ D, et al. Validation of a lateropulsion scale for patients recovering from stroke. Clin Rehabil 2004; 18: 102-109.
12. Baccini M, Paci M and Rinaldi LA. The scale for contraversive pushing: A reliability and validity study. Neurorehabil Neural Repair 2006; 20: 468-472.

13. Krewer C, Riess K, Bergmann J, et al. Immediate effectiveness of single-session therapeutic interventions in pusher behaviour. Gait Posture 2013; 37: 246-250.

14. Karnath HO and Brötz D. Instructions for the Clinical Scale for Contraversive Pushing (SCP). Neurorehabil Neural Repair 2007; 21: 370-371.

15. Clark E, Hill KD and Punt TD. Responsiveness of 2 scales to evaluate lateropulsion or pusher syndrome recovery after stroke. Arch Phys Med Rehabil 2012; 93: 149-155.

16. Babyar SR, White H, Shafi N and Reding M. Outcomes with stroke and lateropulsion: a case-matched controlled study. Neurorehabil Neural Repair 2008; 22: 415-423. 